\title{
AT-rich sequence binding protein 1: Contribution to tumor progression and metastasis of human ovarian carcinoma
}

\author{
JIANGDONG XIANG $^{1 *}$, LINA ZHOU ${ }^{2 *}$, SHUANGDI LI $^{1}$, XIAOWEI XI ${ }^{1}$, JIARONG ZHANG ${ }^{1}$, \\ YANQIU WANG ${ }^{1}$, YIXIA YANG ${ }^{1}$, XUELIAN LIU $^{1}$ and XIAOPING WAN ${ }^{3}$ \\ ${ }^{1}$ Shanghai First People's Hospital, Medical College, Shanghai Jiaotong University, Shanghai 200080; \\ ${ }^{2}$ Shanghai First Maternity and Infant Hospital, Tongji University School of Medicine, Shanghai 200040; \\ ${ }^{3}$ International Peace Maternity and Child Health Hospital, Shanghai 200030, P.R. China
}

Received August 14, 2011; Accepted November 18, 2011

DOI: $10.3892 / \mathrm{ol} .2012 .571$

\begin{abstract}
Special AT-rich sequence binding protein 1 (SATB1) is a master chromatin organizer that has recently been reported to directly upregulate metastasis-associated genes and downregulate tumor suppressor genes. However, its clinical significance in the case of ovarian cancer remains unclear. In the current study, we assessed the expression levels of SATB1 in ovarian cancer and aimed to show whether it may be a conventional clinicopathological parameter. Epithelial ovarian cancer $(n=91)$, borderline cystadenoma $(n=13)$ and normal ovarian background tissues $(n=8)$ were collected immediately following excision during surgery. The mRNA expression levels of SATB1, VEGF-A and MMP-9 were determined using real-time quantitative PCR. Western blotting and immunohistochemical staining were carried out to detect the protein expression levels of SATB1. Expression levels within the ovarian cancer specimens were compared to the normal background tissues and analyzed against FIGO stage, lymph node involvement and histological type. SATB1 mRNA in malignant and borderline ovarian cystadenoma tissues was 6.74- and 5.70-fold higher compared with normal ovarian tissue, respectively $(\mathrm{P}<0.01)$. Western blot analysis revealed that a strong positive band of SATB1 expression was present in ovarian cancer tissues. Immunohistochemical staining showed that the positive expression rates of SATB1 in ovarian cancer, borderline ovarian cystadenoma and normal ovarian tissues were $69.2,61.5$ and $0 \%(\mathrm{P}<0.01)$, respectively. SATB1 expression increased concomitantly with increasing FIGO stage and lymph node involvement. Survival curves showed that a higher SATB1 expression was correlated with shorter survival. Our
\end{abstract}

Correspondence to: Professor Xiaowei Xi, Department of Gynecology and Obstetrics, Shanghai First People's Hospital, Medical College, Shanghai Jiaotong University, No. 100, Haining Road, Shanghai 200080, P.R. China

E-mail: xixiaowei66@yahoo.com.cn

*Contributed equally

Key words: SATB1, ovarian cancer, progression, metastasis, prognosis results provide evidence that SATB1 expression is significantly associated with progression, metastasis and prognosis of epithelial ovarian cancer. SATB1 may therefore serve as a conventional clinicopathological parameter of ovarian cancer.

\section{Introduction}

Ovarian cancer remains the leading cause of mortality from gynecological malignancies (1), due to its insidious symptoms, high incidence of metastasis and high relapse rate. At the time of diagnosis, up to $60 \%$ of cases present at an advanced stage with a low 5-year relative survival rate of approximately $30 \%$ (2) and metastasis has already occurred (3). Lymph node metastasis, which was thought to appear at advanced stages, has been demonstrated to show 10-30\% incidence even at early stages. Patients with negative nodes survived significantly longer than those who had node metastases (4). Therefore, lymphatic metastasis was verified to be a strong indicator of poor prognoses and may account for the majority of ovarian cancer-related deaths (5-9). For the last decade, little was known about the major molecular mechanisms in terms of how genes facilitate metastasis, and this phenomenon is just beginning to be elucidated.

Recently, there has been a marked increase in studies of the activities of special AT-rich sequence binding protein 1 (SATB1) in oncogene regulation. Early on, SATB1 was identified as a 'genome organizer' essential for its crucial role in regulating gene expression during the differentiation and activation of $\mathrm{T}$ cells. However, mounting evidence indicates that as SATB1 is expressed in various types of human cancer, it may be a key protein that controls the development and progression of malignancies (10). At present, tumor growth and metastasis promoted by SATB1 is thought to be a new mechanism of tumor progression. SATB1 exhibits specific epigenetic modifications at target gene loci, directly upregulating metastasis-associated genes while downregulating tumor-suppressor genes. Moreover, investigators found SATB1 to be expressed in a number of other cancer cells, such as gastric cancer, colorectal cancer and laryngeal carcinoma cells, and it appears to be associated with an aggressive phenotype (11-14). Accumulating evidence has shown that SATB1: a) correlates with a high expression of tumor necrosis factor (TNF)- $\alpha$, 
which mediates the inflammatory processes in tumor invasion, angiogenesis and metastasis; b) affects the expression of anti-inflammatory interleukins (IL)-4 and IL-10, which in turn are known to lead to escape from cancer immune surveillance; and c) is significant in anti-apoptotic activity (15-16).

Based on these observations, a hypothesis was generated that SATB1 expression may be a marker used to identify ovarian cancer patients with a high risk of developing metastasis, and it may therefore be a useful prognostic indicator in the clinic. In the current study, we investigated the expression of SATB1 in human ovarian carcinoma cells and explored its potential activities in tumor progression and lymphatic metastasis.

\section{Materials and methods}

Patients and tissues. Prior informed consent for the following studies was obtained from all patients, and the study was approved by the Research Committee for Human Subjects, Shanghai Jiaotong University School of Medicine. A total of 112 clinical samples were obtained between January 2003 and July 2008 from the Pathology Department of Shanghai Jiaotong University Affiliated First People's Hospital, China. Tissue sections included the following categories: 91 cases of epithelial ovarian cancer (25 stage I, 21 stage II and 45 stage III), 13 cases of borderline cystadenoma and 8 cases of normal ovarian tissue. None of the patients had received any adjuvant therapy prior to surgery (neither chemotherapy nor radiotherapy). The samples were collected immediately following surgery. Tissues for RNA isolation were snap-frozen and stored at $-80^{\circ} \mathrm{C}$, and those for immunohistochemistry were fixed in $10 \%$ neutral-buffered formalin and embedded in paraffin. The clinical backgrounds of ovarian cancer were evaluated by the International Federation of Gynecology and Obstetrics (FIGO, 2000) classification. Patients were treated and followed-up at the Shanghai Jiao Tong University Affiliated First People's Hospital, China to ensure collection of clinical data.

$Q T-P C R$. The mRNA expression levels of SATB1, VEGF-A and MMP-9 were measured by quantitative real-time PCR (QT-PCR). Total RNA was extracted from tumor tissues with TRIzol reagent according to the manufacturer's instructions. $\beta$-actin mRNA served as an internal standard. The primers used were designed based on information from the human genomic database. The primers used were SATB1 (Gene ID: 6304): 5'-TGCAAAGGTTGCAGCAACCAAAAGC-3' (sense) and 5'-AACATGGATAATGTGGGGCGGCCT-3' (antisense); VEGF-A (Gene ID: 7422): 5'-TGCTTCTGAGTT GCCCAGGA-3' (sense) and 5'-TGGTTTCAATGGTGT GAGGACATAG-3' (antisense); MMP-9 (Gene ID: 4318): 5'-ACGCACGACGTCTTCCAGTA-3' (sense) and 5'-CCACC TGGTTCAACTCACTCC-3' (antisense); primers used to amplify $\beta$-actin were: 5'-TGGCACCCAGCACAATGAA-3' (sense) and 5'-CTAAGTCATAGTCCGCCTAGAAGCA-3' (antisense). Each sample was tested in triplicate and the threshold cycle $(\mathrm{Ct})$ for each target mRNA was normalized to $\beta$-actin mRNA and averaged using the formula $\left({ }_{\Delta} \mathrm{Ct}\right.$, $\left.{ }_{\Delta} \mathrm{Ct}={ }_{\Delta \mathrm{CTSATB} 1 / \text { VEGF-A/MMP-9}}{ }^{-} \mathrm{Ct}_{\beta \text {-actin }}\right)$. Inversed $\left({ }_{\Delta \Delta} \mathrm{Ct}\right)$ values were used for the statistical analyses. Relative abundance of mRNA levels was determined using the formula $2^{-\Delta \Delta \mathrm{Ct}}(17)$.
Western blot analyses. Cellular protein was quantitated by Bradford assay (Bio-Rad), and $50 \mu \mathrm{g}$ of the cleared lysates were separated on a $12 \%$ SDS-PAGE, and electro-transferred onto PVDF membranes (Amersham Pharmacia Biotech). GAPDH was used as an equal loading control. PVDF (Millipore, Bedford, MA, USA) membranes were blocked in Tris-buffered saline containing $0.1 \%$ Tween-20 (TBST) with 5\% non-fat dry milk for $2 \mathrm{~h}$, and incubated with a polyclonal rabbit anti-human SATB1 antibody (Abcam). The membranes were then washed 3 times for $5 \mathrm{~min}$ in PBST, followed by incubation with horseradish peroxidase-conjugated goat anti-rabbit antibody (Abcam) in PBST for $1 \mathrm{~h}$. After washing 3 times with PBST, the bands were developed using the enhanced chemiluminescence (ECL) detection system (Pierce Biotech Inc., Rockford, IL, USA) according to the manufacturer's instructions.

Immunohistochemical staining. Immunohistochemical staining was performed on samples fixed in $10 \%$ neutralbuffered formalin and embedded in paraffin. Paraffin sections $(5 \mu \mathrm{m})$ were dewaxed in xylenes and rehydrated in serial dilutions of ethanol. Antigen retrieval was carried out by heating to $120^{\circ} \mathrm{C}$ in $10 \mathrm{mM}$ citrate buffer solution ( $\mathrm{pH} 6.0$ ). Endogenous peroxidase activity was blocked by $3 \% \mathrm{H}_{2} \mathrm{O}_{2}$ for $10 \mathrm{~min}$ at room temperature, and non-specific binding of reagents was quenched by $10 \%$ normal goat serum for $20 \mathrm{~min}$ at $37^{\circ} \mathrm{C}$. Sections were then incubated with the primary antibody [polyclonal rabbit anti-human SATB1 (diluted 1:150, Abcam)] overnight in a moist chamber at $4^{\circ} \mathrm{C}$. Following washing in phosphate-buffered saline (PBS), slices were incubated with the appropriate secondary antibodies (biotinylated goat anti-rabbit IgG) and then streptavidin-biotin-horseradish peroxidase complex at $37^{\circ} \mathrm{C}$, respectively. The immunoreactions were developed by diaminobenzidine (DAB) solution, and the crimson yellow precipitates were identified as positive staining. Counterstaining was performed with ameliorative Gill's hematoxylin and slides were dehydrated and mounted. Immunohistochemical staining was evaluated by a semi-quantitative method according to the intensity and percentage of cells with SATB1 nuclear staining on the following scale: score 0 , negative nuclear staining for all tumor cells; score 1 , weak nuclear staining representing all positive staining other than score 2; score 2, moderate nuclear staining for $>50 \%$ or strong staining for $>5 \%$ of the tumor cells. This scoring system was previously validated (10).

Statistical analysis. Results were presented as the means \pm standard deviation (SD). One-way analysis of variance (ANOVA) was used to determine statistical significance in the comparisons of SATB1, VEGF-D and MMP-9 expression levels among the different groups. Overall survival analyses were performed using the Kaplan-Meier method. Multivariate analysis of prognostic factors was performed using Cox's regression model. $\mathrm{P}<0.05$ was considered to indicate a statistically significant difference.

\section{Results}

Overexpression of SATB1 in ovarian cancer cells. The expression levels of SATB1 in different tissues were determined by QT-PCR and western blotting assay. QT-PCR confirmed that 


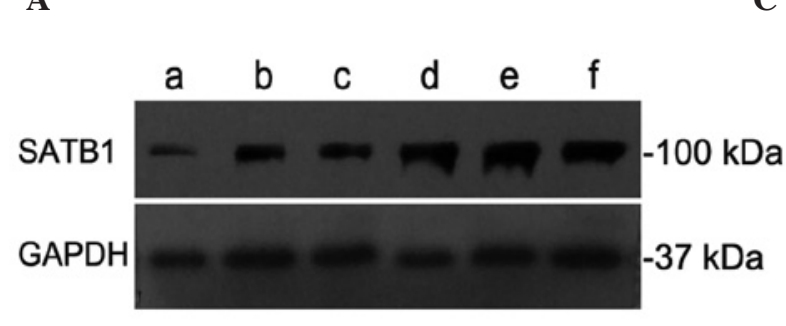

B

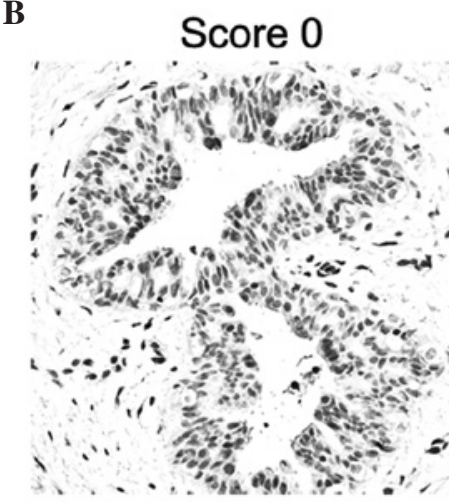

C

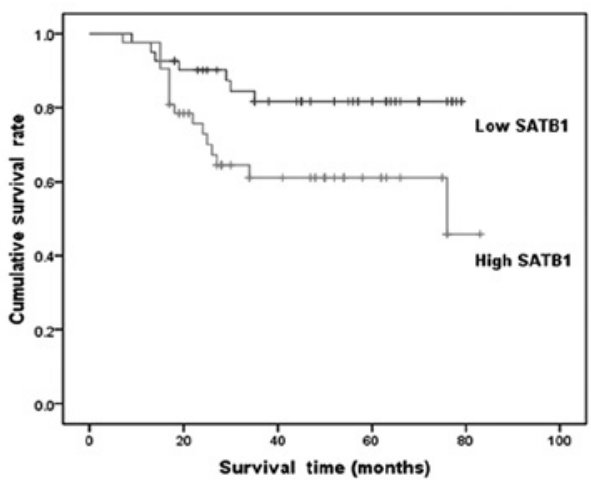

Score 2

Figure 1. Western blot assay, immunohistochemical staining and overall survival (OS) curve. (A) Western blotting assay showed SATB1 expression levels in different FIGO stage ovarian cancer cells. FIGO stage I (lane a), II (lanes b and c) and III (lanes d, e and f) exhibited the same positive band at approximately $37 \mathrm{kDa}$ (GAPDH); stage III (lanes d, e and f) showed a strong positive band at approximately $100 \mathrm{kDa}$ (SATB1); stage II (lanes b and c) showed a moderate positive band, but stage I (lane a) showed a weak or negative band. (B) Immunohistochemical staining showed a score 0 , score 1 and score 2 staining of SATB1 in ovarian cancer tissues (score explained in Materials and methods). Results were assessed on the basis of intensity and the proportion of positive-staining cells. The positive staining of tumor cells was expressed as yellow-brown particles in the nuclei. (C) Survival curve of 91 patients with ovarian carcinomas shows that a higher expression of SATB1 was associated with lower OS (P<0.05). FIGO, International Federation of Gynecology and Obstetrics.

SATB1 had higher mRNA expression levels in malignant cells at 6.74-fold, and in borderline cystadenoma cells at 5.70-fold higher than in normal cells $(\mathrm{P}<0.05)$. Western blotting showed a concomitant increase in the expression level of SATB1 with the increased FIGO stage. GAPDH was used as an equal loading control. FIGO stage I (lane a), stage II (lanes b and c) and stage III (lanes d, e and f) showed the same positive band at $\sim 37 \mathrm{kDa}$ (GAPDH); stage III (lanes d, e and f) showed a strong positive band at $\sim 100 \mathrm{kDa}$ (SATB1); stage II (lanes b and c) showed a moderate positive band, but stage I (lane F) showed a weak or negative band (Fig. 1A). These results indicate that SATB1 may promote tumor progression in ovarian cancer.

Strong positive nuclei in ovarian cancer tissues. Immunohistochemical staining revealed a number of strong SATB1-positive nuclei in ovarian cancer tissues (dark brown nuclei), suggesting that SATB1 may exhibit marked activities in ovarian tumor cell progression (Fig. 1B). Immunostaining was evaluated by a semi-quantitative method according to the intensity and percentage of cells with SATB1 nuclear staining as described previously in Materials and methods. The result showed that 25 ovarian cancer samples received a score of 2 , 38 samples received a score of 1 , and 28 samples received a score of 0 . Therefore, in this study, the positive expression rate of SATB1 in ovarian cancer tissues was $69.2 \%$. This result provides evidence that SATB1 may contribute markedly to ovarian cancer progression.
SATB1 decreased the survival time. The effect of SATB1 on ovarian cancer was also reflected in the overall survival (OS) time. Survival of the patients who had negative or low expression of SATB1 was markedly prolonged compared with the positive or high expression patients. Higher expression levels showed a significant association with poorer OS. Statistical significance in OS was observed between low and high expression levels of SATB1 ( $\mathrm{P}<0.05$, Fig. 1C).

Relationship between SATB1 mRNA expression and the clinicopathological parameters. To address the question of the relevance of SATB1 on human ovarian cancer histological type, differentiation degree, FIGO stage and lymphatic metastasis, we compared SATB1 mRNA levels among different parameters, respectively (Table I). Results of this study indicate that SATB1 expression increased concomitantly with FIGO stage, and significant differences were observed between stages I, II and III $(\mathrm{P}<0.01)$. Furthermore, a strong nucleus SATB1 expression was detected in the majority of the malignant ovarian tissues with lymphatic metastasis. No difference was observed between various histological types and differentiation categories $(\mathrm{P}>0.05)$. Therefore, $\mathrm{SATB} 1$ contributes significantly to ovarian cancer progression and metastasis.

Overexpression of metastatic relevant molecules in ovarian cancer tissues. QT-PCR showed that higher expression levels of VEGF-A and MMP-9 were identified in malignant ovarian 
Table I. Relationship between SATB1 mRNA expression in epithelial ovarian cancer and the clinicopathological parameters.

\begin{tabular}{lrcr}
\hline & $\mathrm{n}$ & $\begin{array}{c}2^{-\Delta \Delta \mathrm{Ct}} \\
\text { (relative multiple) }\end{array}$ & P-value \\
\hline Histological type & & & 0.053 \\
Serous & 50 & $7.21 \pm 2.03$ & \\
Mucinous & 10 & $8.51 \pm 2.79$ & \\
Endometrioid & 9 & $6.12 \pm 2.03$ & \\
Clear cell & 18 & $5.75 \pm 2.47$ & \\
Undifferentiated & 4 & $7.57 \pm 2.73$ & \\
Differentiation & & & \\
Poor & 27 & $7.38 \pm 2.17$ & 0.000 \\
Moderate & 28 & $7.08 \pm 2.09$ & \\
Well & 18 & $7.42 \pm 2.57$ & \\
Stage & & & 0.001 \\
I & 25 & $5.31 \pm 1.66$ & \\
II & 21 & $6.67 \pm 2.67$ & \\
III & 45 & $8.04 \pm 1.92$ & \\
Lymphatic metastasis & & & \\
Negative & 56 & $6.37 \pm 2.46$ & \\
Positive & 35 & $7.94 \pm 1.78$ & \\
\hline
\end{tabular}

QT-PCR was performed to detect the mRNA expression levels of SATB1 in different clinicopathological parameters. The results show a concomitant increase in SATB1 expression with the increased FIGO stage and nodal involvement in ovarian carcinomas $(\mathrm{P}<0.05)$. However, no significant difference is observed in different histological types and differentiation degrees ( $\mathrm{P}>0.05)$. QT-PCR, quantitative real-time PCR; FIGO, International Federation of Gynecology and Obstetrics.

cancer and borderline cystadenoma tissues, which exhibited extensive invasions and metastasis to remote multistep lymph nodes and organs. The results show that VEGF-A expression in ovarian cancer and borderline cystadenoma tissues was 17.49- and 12.48-fold higher than in normal cells, respectively. MMP-9 expression showed 11.86- and 7.69-fold higher levels than normal cells in ovarian cancer and borderline cystadenoma tissues, respectively. The present study also indicated a relationship between VEGF-A/MMP-9 mRNA expression levels and ovarian cancer histological type, differentiation degree, FIGO stage and lymphatic metastasis. Our data showed that significant differences of VEGF-A and MMP-9 expression were observed at various differentiation degrees, FIGO stages and lymphatic metastasis (Fig. 2), but not in different histological types.

\section{Discussion}

Epithelial ovarian cancers are the most serious threat to the health of women (18), due to the high incidence of metastasis and low 5-year survival rate. Clinical and pathological observations have shown that lymph node involvement is one of the earliest features of metastatic disease, and this observation holds true for the majority of ovarian cancers. Metastasis appears to be the most lethal attribute of malignant tumors and the major cause of cancer morbidity and mortality $(19,20)$. Currently, gene regulation mechanisms in tumor progression and metastases have become a hot spot in oncology research. Therefore, emphasis has been placed on finding potential genes that indicate metastatic probability and poor prognosis of malignancies. These genes may become promising therapeutic targets for clinical diagnosis and therapies.

SATB1 was shown to act as a new type of gene regulator by selectively tethering far distal specialized DNA sequences to its cage-like network, and assembling them with chromatin modifying and transcription factors to ensure the accurate expression of its target genes (21-23). SATB-1 has been proposed to act as a determinant for the acquisition of further aggressive phenotypes, characterized by enhanced metastatic and invasive potential. Investigators have indicated that SATB1 is not expressed in all cells and it appears particularly crucial in cells that change their function, as numerous progenitor cells do, including the thymocytes that turn into T cells, and as cancer cells do to turn into metastatic cells (10). Studies have shown that a variety of genes involved in tumorigenesis are regulated by SATB1, indicating a plethora of SATB1-targeted genes that collectively induce tumor growth and metastasis (10). Moreover, by introducing SATB1 into otherwise non-metastatic breast cancer cells, invasive tumors may be induced in mice; conversely, removing SATB1 from metastatic cells may markedly reduce the invasive capacity of these cells, and inhibit or regress tumor growth. Therefore, we hypothesized that SATB1 expression is also detected in ovarian cancer cells, and SATB1 may be an essential contribu-
A

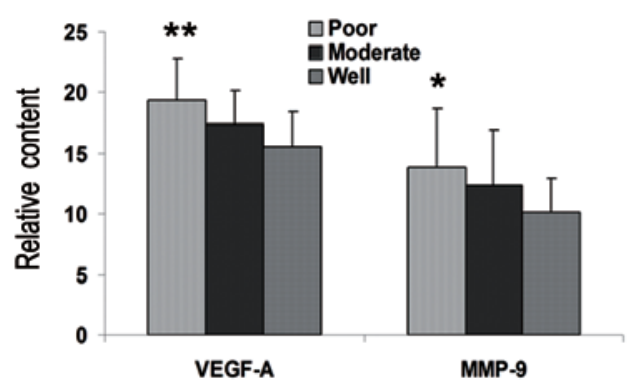

B

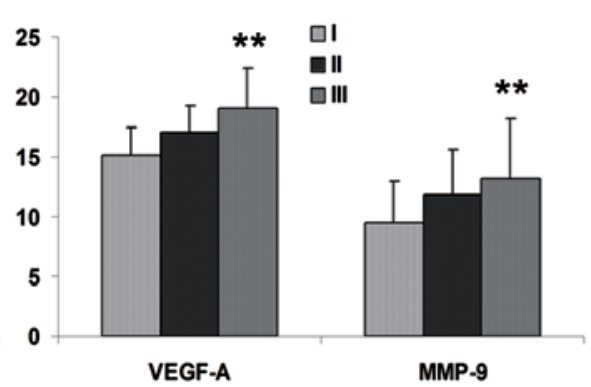

C

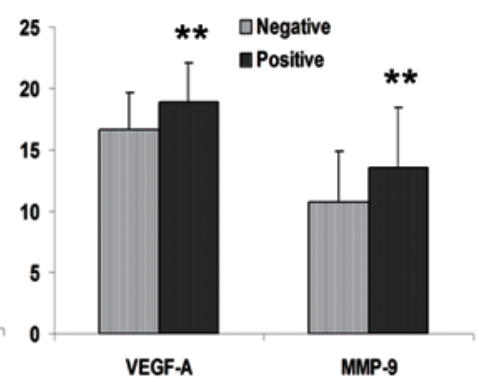

Figure 2. Quantitative real-time PCR (QT-PCR). QT-PCR showed expression levels of VEGF-A and MMP-9 in ovarian cancer tissues. The expression levels were concomitant with the increased (A) differentiation degree, (B) FIGO stage and (C) nodal involvement in ovarian carcinomas $\left({ }^{*} \mathrm{P}<0.05 ;{ }^{* *} \mathrm{P}<0.01\right)$. VEGF, vascular endothelial growth factor-A; MMP-9, matrix metalloproteinase-9; FIGO, International Federation of Gynecology and Obstetrics. 
ting factor in the multiphase evolution from a benign to an invasive malignant ovarian cancer.

The present study explored the potential of the SATB1 protein as a conventional clinicopathological parameter for human ovarian cancer. We found SATB1 expression to be upregulated in malignant ovarian cancer cells that exhibit a higher invasive potential than the parental cells. Our data revealed that an increasing SATB1 expression was concomitant with an increasing stage of the invasive ovarian carcinoma and decreasing OS. Therefore, SATB1 expression is a marker that can be used to identify ovarian cancer patients with a high risk of developing metastases, and it may become a useful prognostic marker in the clinic. Investigators have shown that the SATB1 level in the nuclei of breast cancer cells has a high prognostic significance and a high level of SATB1 expression is an excellent indicator of poor prognosis. SATB1 has previously been proposed as a key protein that controls the development and progression of breast cancer. Similar results may be observed in our study: the highest levels of SATB1 were in samples from patients whose survival times were the shortest, whereas patients whose tumor samples had a weak or negative SATB1 expression generally had longer survival times. The OS curve showed that a higher expression of SATB1 was markedly correlated with a shorter OS time; therefore, we may conclude the same effects of SATB1 in ovarian cancer. Scoring on the basis of the percentage of SATB1-positive cells indicated that SATB1 expression was associated significantly with FIGO stage and nodal involvement in ovarian epithelial carcinoma patients. A higher SATB1 expression was correlated with worse prognosis. These encouraging results suggest that SATB1 expression may be the probable progression and metastatic mechanism of ovarian cancer.

Accumulating evidence indicates that VEGF plays a key role in vascular permeability, angiogenesis, lymphangiogenesis and endothelial cell growth, and induces tumor metastasis (24). Furthermore, the upregulation of MMP-9 promotes tumor invasion and migration $(25,26)$. In metastatic breast cancer cells, SATB1 reprograms the genome to change the expression of hundreds of genes affecting cell adhesion, cell signaling, cellcycle regulation and other functions, promoting tumor growth and metastasis (10). In the present study, SATB1 was found to be highly expressed in malignant ovarian carcinoma cells and tissues with VEGF-A and MMP-9 overexpression. Therefore, we hypothesize that the expression of VEGF-A and MMP-9, which has significant functions in promoting metastasis, may be affected by SATB1 in ovarian cancer. Additionally, the detailed relationships are to be clarified in further studies. To the best of our knowledge, this is the first report that not only combined SATB1 with poor clinical outcomes, but also correlated it with short OS and a high expression of VEGF-A and MMP-9 in epithelial ovarian cancer.

Our results suggest that SATB1 expression increased concomitantly with tumor grade and lymph node status; higher expression of SATB1 in malignant ovarian cancer cells was correlated with a lower survival rate. Therefore, SATB1 appears to be a novel independent prognostic marker for poor clinical outcomes in epithelial ovarian carcinoma.

Vascular endothelial growth factor-A (VEGF-A) and matrix metalloproteinase-9 (MMP-9), which are closely associated with tumor invasion and metastasis, were also detected in our study. Our results showed that ovarian cancer tissues exhibited a strong expression of VEGF-A and MMP-9; nevertheless, normal ovarian tissue had a weak or negative expression. We hypothesized that there may be a crucial regulatory relationship between SATB1 and VEGF-A/MMP-9 expression during the formation of tumor metastasis in ovarian carcinoma, such as the similar upregulation effect reported by Han et al in breast cancer (10).

In conclusion, our data provide significant evidence on the relationships between a positive SATB1 expression and high FIGO stage, lymph node metastases and low survival rates in ovarian cancer. SATB1 may therefore be a conventional clinicopathological parameter in ovarian cancer. However, future studies are required to elucidate the precise mechanisms by which SATB1 promotes the progression and metastasis of ovarian carcinomas.

\section{Acknowledgements}

We thank Yinyan He for her technical help. This study was supported by the Project of Shanghai Science and Technology Commission (No. 07JC14031).

\section{References}

1. Partridge E, Kreimer AR, Greenlee RT, et al: Results from four rounds of ovarian cancer screening in a randomized trial. Obstet Gynecol 113: 775-782, 2009.

2. Jemal A, Siegel R, Ward E, Hao Y, Xu J, Murray T and Thun MJ: Cancer statistics, 2008. CA Cancer J Clin 58: 71-96, 2008.

3. Tingulstad S, Skjeldestad FE, Halvorsen TB and Hagen B: Survival and prognostic factors in patients with ovarian cancer. Obstet Gynecol 101: 885-891, 2003.

4. Balbi G, Manganaro MA, Monteverde A, Landino I, Franzese C and Gioia F: Ovarian cancer: lymph node metastases. Eur J Gynaecol Oncol 30: 289-291, 2009.

5. Brewer MA, Johnson K, Follen M, Gershenson D and Bast R: Prevention of ovarian cancer: intraepithelial neoplasia. Clin Cancer Res 9: 20-30, 2003.

6. Concin N, Hefler L, Van Bavel J, et al: Biological markers in pT1 and pT2 ovarian cancer with lymph node metastases. Gynecol Oncol 89: 9-15, 2003.

7. Jemal A, Tiwari RC, Murray T, et al: Cancer statistics, 2004. CA Cancer J Clin 54: 9-29, 2004.

8. Takeshima N, Hirai Y,Umayahara K,Fujiwara K, Takizawa K and Hasumi K: Lymph node metastasis in ovarian cancer: difference between serous and non-serous primary tumors. Gynecol Oncol 99: 427-431, 2005.

9. Ayhan A, Gultekin M, Taskiran C, Celik NY, Usubutun A, Kucukali $\mathrm{T}$ and Yuce K: Lymphatic metastasis in epithelial ovarian carcinoma with respect to clinicopathological variables. Gynecol Oncol 97: 400-404, 2005.

10. Han HJ, Russo J, Kohwi Y and Kohwi-Shigematsu T: SATB1 reprogrammes gene expression to promote breast tumor growth and metastasis. Nature 452: 187-193, 2008.

11. Lu X, Cheng C, Zhu S, et al: SATB1 is an independent prognostic marker for gastric cancer in a Chinese population. Oncol Rep 24: 981-987, 2010.

12. Rygiel AM, Davelaar AL, Milano F, Fockens $P$ and Krishnadath KK: SATB1 influences expression of cytokines in colorectal cell lines and is associated with a more aggressive phenotype. AGA abs. S2050, 2009.

13. Agrelo R, Souabni A, Novatchkova M, et al: SATB1 defines the developmental context for gene silencing by Xist in lymphoma and embryonic cells. Dev Cell 16: 507-516, 2009.

14. Zhao XD, Ji WY, Zhang W, He LX, Yang J, Liang HJ and Wang LL: Overexpression of SATB1 in laryngeal squamous cell carcinoma. ORL J Otorhinolaryngol Relat Spec 72: 1-5, 2010

15. Gotzmann J, Meissner M and Gerner C: The fate of the nuclear matrix-associated-region-binding protein SATB1 during apoptosis. Cell Death Differ 7: 425-438, 2000. 
16. Gong F, Sun L, Wang Z, et al: The BCL2 gene is regulated by a special AT-rich sequence binding protein 1-mediated long range chromosomal interaction between the promoter and the distal element located within the 3'-UTR. Nucleic Acids Res 39: 4640-4652, 2011.

17. Livak KJ and Schmittgen TD: Analysis of relative gene expression data using real-time quantitative PCR and the 2(-delta delta C(T)) method. Methods 25: 402-408, 2001.

18. Harlan LC, Clegg LX and Trimble EL: Trends in surgery and chemotherapy for women diagnosed with ovarian cancer in the United States. J Clin Oncol 21: 3488-3494, 2003.

19. Yoshida BA, Sokoloff MM, Welch DR and Rinker-Schaeffer CW: Metastasis-suppressor genes: a riview and perspective on an emerging field. J Natl Cancer Inst 92: 1717-1730, 2000.

20. Cheung ST, Chen X, Guan XY, et al: Identify metastasis-associated genes in hepatocellular carcinoma through clonality delineation for multinodular tumor. Cancer Res 62: 4711-4721, 2002.
21. Cai S, Han HJ and Kohwi-Shigematsu T: Tissue-specific nuclear architecture and gene expression regulated by SATB1. Nat Genet 34: 42-51, 2003.

22. Cai S, Lee CC and Kohwi-Shigematsu T: SATB1 packages densely looped, transcriptionally active chromatin for coordinated expression of cytokine genes. Nat Genet 38: 1278-1288, 2006.

23. Yamaguchi H, Tateno $M$ and Yamasaki K: Solution structure and DNA-binding mode of the matrix attachment region-binding domain of the transcription factor SATB1 that regulates the T-cell maturation. J Biol Chem 281: 5319-5327, 2006.

24. Ellis LM: Epidermal growth factor receptor in tumor angiogenesis. Hematol Oncol Clin North Am 18: 1007-1021, 2004.

25. Hung WC, Tseng WL, Shiea J and Chang HC: Skp2 overexpression increases the expression of MMP-2 and MMP-9 and invasion of lung cancer cells. Cancer Lett 288: 156-161, 2010.

26. Hao L, Zhang C, Qiu Y, et al: Recombination of CXCR4, VEGF, and MMP-9 predicting lymph node metastasis in human breast cancer. Cancer Lett 253: 34-42, 2007. 